\title{
Relationships between upper-limb functional limitation and self-reported disability 3 months after stroke
}

\author{
Alexander W. Dromerick, MD; ${ }^{1-3^{*}}$ Catherine E. Lang, PhD, PT; ${ }^{3}$ Rebecca Birkenmeier, MS, OTR; ${ }^{1}$ \\ Michele G. Hahn, MS, OTR; ${ }^{2}$ Shirley A. Sahrmann, PhD, PT; ${ }^{1,3}$ Dorothy F. Edwards, PhD ${ }^{1-2}$ \\ ${ }^{1}$ Department of Neurology, and Programs in ${ }^{2}$ Occupational Therapy and ${ }^{3}$ Physical Therapy, Washington University, \\ St. Louis, $M O$
}

\begin{abstract}
This study explored relationships between upperlimb (UL) functional limitations and self-reported disability in stroke patients with relatively pure motor hemiparesis who were enrolled in an acute rehabilitation treatment trial. All participants were enrolled in the VECTORS (Very Early Constraint Treatment for Recovery from Stroke) study. VECTORS is a singlecenter pilot clinical trial of early application of constraintinduced movement therapy (CIMT). All 39 subjects who completed 90 days of VECTORS were included in this analysis. Trained study personnel who were blinded to the treatment type performed all evaluations. Data in this article examine relationships between assessments performed 90 days after stroke. Functional limitation measures included the Action Research Arm (ARA) test and Wolf Motor Function Test (WMFT), and self-reported disability measures included the Functional Independence Measure (FIM) and Motor Activity Log (MAL) (by telephone). Mean plus or minus standard deviation time from stroke onset to randomization was 9.4 plus or minus 4.3 days, and median time to follow-up was 99 days (range 68-178). Subjects with perfect or near-perfect scores on the ARA test or WMFT reported residual disability on the FIM and MAL. Quality of movement on the WMFT (functional ability score) was not strongly associated with self-reported frequency, and speed of movement on the WMFT (timed score) was not associated with self-reported frequency (MAL amount of use). In this early UL intervention trial, we found that perceived disability measures captured information that was not assessed by functional limitation and impairment scales. Our results indicate that excellent motor recovery as measured by functional limitation and impairment scales did not equal restoration of everyday productive UL use and speed of task completion did not translate to actual use. Our results confirm the need for a measurement strategy that is sensitive to change, assesses a broad performance range, and
\end{abstract}

detects meaningful clinical improvements in early rehabilitation intervention trials.

Key words: activities of daily living, arm, cerebrovascular accident, constraint therapy, functional limitation, hemiplegia, motor skills, outcome assessment (healthcare), randomized controlled trials, rehabilitation, upper limb.

\section{INTRODUCTION}

Persistent loss of upper-limb (UL) motor function is found in 45 percent of all stroke survivors and contributes substantially to stroke-related disability [1]. Current rehabilitation care focuses on return to independence in basic and instrumental activities of daily living (ADL) rather than on restoration of motor or sensory function.

Abbreviations: $\mathrm{ADL}=$ activities of daily living, $\mathrm{AOU}=$ amount of use, ARA = Action Research Arm (test), CIMT = constraintinduced movement therapy, FIM = Functional Independence Measure, MAL $=$ Motor Activity Log, NIHSS $=$ National Institutes of Health Stroke Scale, QOM = quality of movement, UL = upper limb, VECTORS = Very Early Constraint Treatment for Recovery from Stroke, WMFT = Wolf Motor Function Test.

${ }^{*}$ Address all correspondence to Alexander Dromerick, MD; National Rehabilitation Hospital, 102 Irving Street NW, Washington, DC 20010-2949; 202-877-1932; fax: 202726-7521. Email: alexander.w.dromerick@medstar.net DOI: 10.1682/JRRD.2005.04.0075 
More recently, treatments are being proposed based on animal and human data that describe how the brain reacts to injury and how various training paradigms alter or improve motor recovery. These treatments, including functional electrical stimulation [2], constraint-induced movement therapy (CIMT) [3-5], and robotic training [6], are all employed with the explicit goal of improving UL motor function. Trials of these treatments will require researchers to use a practical, sensitive, and valid measurement strategy to determine to what extent any given treatment is effective and whether one treatment may be superior to another.

Underlying the proposed rehabilitation techniques is the assumption that improvements in UL motor function will lead to reduced disability and increased social participation. Consequently, studies of newer techniques often use a functional limitation measure as the primary end point and only secondarily assess disability and participation. Yet, the ultimate goal of any treatment is to promote independence and productive activities. Improved UL motor function may then be viewed as simply the means to this ultimate goal.

Unfortunately, standardized measures of UL functional limitations may not adequately predict actual UL use for ADL [1,7]. At this time, relationships between UL motor performance as seen in the laboratory or clinic and actual UL use in everyday life are poorly understood, particularly in the context of acute rehabilitation treatment trials. This presents a methodological challenge, since improvement on a UL functional limitation measure cannot simply be assumed to translate to increased everyday UL use, reduced disability, or improved participation.

While the relationships between UL functional limitation and associated disability have been extensively explored in populations with subacute and chronic stroke [8-9], they have not been fully examined in an acute inpatient rehabilitation cohort. The population we have chosen is of particular interest to investigators because similar populations are used in clinical trials of early motor interventions and in studies of early motor recovery. The first 90 days after stroke may be critical for the application of behavioral interventions such as CIMT because this may be when patients are most responsive to such treatments. Several authors suggest that earlier rehabilitation interventions may be more effective [7,10-11]. Moreover, recent laboratory data suggest that rodents respond best to motor interventions initiated within 14 days of injury and not at all if motor training is delayed until 30 days after injury [12]. If earlier intervention proves effective, investigators will be challenged to develop a measurement strategy that will be sensitive to change, will assess a broad range of performance, and will detect meaningful clinical improvements.

Our specific goal in this analysis was to evaluate the relationships among the impairment, functional limitation, and perceived disability assessments used in the Very Early Constraint Treatment for Recovery from Stroke (VECTORS) clinical trial. The outcome of the VECTORS trial and issues regarding CIMT will be discussed in other articles once the trial is completed and the data are unblinded. At the time of this writing, the study was ongoing.

\section{METHODS}

\section{Subjects}

All participants were enrolled in the VECTORS study. The Washington University Human Studies Committee approved the study, and all subjects provided informed consent before participation. VECTORS is a single-center pilot clinical trial of the early application of CIMT; through VECTORS, necessary information is gathered for the design of a definitive multicenter trial of CIMT administered in the immediate poststroke period. Study and treatment procedures are described elsewhere [4]. VECTORS has a randomized, single-blind, controlled design. Subjects were randomized to one of three groups: (1) 2 hours a day of conventional treatment, (2) 2 hours a day of shaping treatment plus 6 hours of CIMT, or (3) 3 hours a day of shaping plus CIMT 90 percent of waking hours.

Thirty-nine subjects who had completed 90 days of VECTORS study participation were included in this study. Inclusion and exclusion criteria were intended for the selection of a group of hemiparetic subjects with some residual voluntary UL control and limited sensory or cognitive impairments who required inpatient rehabilitation and were unlikely to have complete, spontaneous recovery. Subjects were included if they had-

1. An ischemic or hemorrhagic stroke (with confirmatory neuroimaging) within 28 days of admission to inpatient rehabilitation.

2. Persistent hemiparesis indicated by a score of 1 to 3 (where 1 designates a downward drift of the extended arm, 2 designates some effort against gravity, and 3 designates no effort against gravity) on the motor arm 
item of the National Institutes of Health Stroke Scale (NIHSS) [13].

3. Some proximal UL voluntary activity indicated by a score of 3 or higher on the UL item of the Motor Assessment Scale [14]; the ability to move the wrist and fingers was not required.

4. Evidence of preserved cognitive function indicated by a score of 0 or 1 on the consciousness and communication items of the NIHSS, the ability to perform two-step commands, and a score of 8 or lower on the Short Blessed Memory Orientation and Concentration Scale [15].

5. No UL injury or conditions that limited use before the stroke.

Subjects were excluded if they-

1. Could not give informed consent.

2. Had clinically significant fluctuations in mental status in the 72 hours before enrollment.

3 . Were not independent before the index stroke as measured by scores $<95$ on the Barthel Index [16] or $>1$ on the Modified Rankin Scale [17].

4. Had hemispatial neglect determined by more than three errors on the Star Cancellation Test.

5. Had severe sensory loss determined by a score of 2 on the sensory item of the NIHSS.

6. Were not expected to survive 1 year because of other illnesses (e.g., cardiac disease, malignancy).

\section{Procedures}

Trained study personnel who were blinded to the treatment type performed all evaluations. The data in this article focus on the assessments performed at 90 days after stroke onset. The 90-day time point was chosen as the primary study end point because most acute stroke intervention trials assess efficacy at 90 days after onset, when the majority of stroke patients are at or near their clinical plateau [18].

\section{Measures}

\section{Functional Limitation Measures}

Action Research Arm Test. The Action Research Arm (ARA) test assesses functional limitations of the UL on the affected side. It includes 19 items divided into four subscales: grasp, grip, pinch, and gross movement. Reliability (interrater $=0.99$, test-retest $=0.98$ ), construct validity, and predictive validity of the ARA test have been well established [19-20]. The ARA test uses ordinal scoring for each subtest item. A score of 0 indicates no movement and a score of 3 indicates normal movement. Item scores are summed to create subtest and full-scale scores, with a maximum score of 57 indicating normal performance.

Wolf Motor Function Test. The Wolf Motor Function Test (WMFT) is a 17-item measure used for quantification of UL functional limitations. It comprises 2 strength items and 15 timed-task performance items. The task performance items begin with measuring simple proximal movements and progress to more complex distal and whole limb movements. The WMFT yields two scores: a functional ability score, quantifying quality of performance, and a timed score, quantifying speed of performance in seconds. Reliability and validity of this test have been well established [21-23]. In the VECTORS study, the "key use" task was not collected, and the results reported do not include this item.

\section{Disability Measures}

Motor Activity Log. The Motor Activity Log (MAL) is a focal measure of self-perceived disability on tasks requiring UL use. It is made up of a semistructured interview for assessing how much and how well patients use their affected arm for ADL in the home over a specified period of time [9]. Thirty specific ADL tasks are evaluated with the use of a 6-point amount of use (AOU) scale and a 6-point quality of movement (QOM) scale. The tasks include buttoning a shirt, brushing teeth, and using a key. The MAL is administered independently to the patient and a significant other or informant. Adequate interrater reliability and internal consistency have been reported. Recently, the validity of a 14 -item version has been demonstrated [24]. The self-report of study participants is reported in this article.

Functional Independence Measure. The Functional Independence Measure (FIM) is an 18-item global disability measure that incorporates concepts and items of functional performance including ADL, bowel and bladder function, social cognition, functional communication, and functional mobility [25]. The FIM is scored on a 7-point ordinal scale in which a score of 1 indicates dependence and a score of 7 indicates independence. Reliability and validity of the FIM have been established. Interrater reliability is 0.95 [26]. Construct validity for individuals surviving stroke is $r=0.65$ ( $r=$ Pearson's product moment correlation coefficient) [27]. The FIM is endorsed by the Agency for Healthcare. All evaluators in this study are 
certified by the Uniform Data System. In this study, the FIM was scored by telephone interview and is therefore considered a measure of self-perceived disability.

\section{Impairment Measure}

The NIHSS assesses cognitive, sensory, and motor impairments to indicate overall stroke severity [13]. This 13-item test results in scores ranging from 0 (no deficit) to 46 (severe deficit). Scores of 6 through 20 are widely considered to be in the moderate range. The NIHSS scores reported here were collected during the acute hospital service; this is the only measure reported in this article from the prerandomization assessment.

\section{Analysis}

We conducted statistical analyses using Statistical Analysis System for Windows, version 9, SAS Institute Inc, Cary, North Carolina. Descriptive statistics were calculated for all variables, and the normality of distribution was examined for all study variables. We used contingency tables and Student's $t$-tests with Bonferroni corrections for multiple comparisons to compare groups for normally distributed variables. Wilcoxon Signed Rank Tests were used for comparisons between hemorrhagic and ischemic strokes because of the unequal sample sizes. Pearson's product moment correlation coefficients ( $r$ ) were computed to examine relationships among the measures.

\section{RESULTS}

At the Barnes-Jewish Hospital in St. Louis, Missouri, 1,117 persons admitted to the acute neurology stroke service were screened for study eligibility through October 2004. Of these, 48 individuals gave their informed consent and were randomized into the VECTORS study; 90-day assessments were available on 39 subjects at the time of this writing. Mean time \pm standard deviation to randomization was $9.4 \pm 4.3$ days. Median time to follow-up was 99 days (range 68-178). Table 1 presents the demographic characteristics of the 39 subjects for whom the data were available at the time of this study. Subjects were enrolled based on the presence of a moderate degree of UL motor dysfunction and the absence of severe sensory or cognitive impairments. Study participants displayed a wide range of overall stroke severity as measured by the total NIHSS. Similarly, because no age criteria were used for study eligibility, subjects ranged in age from 39 to 94 years. Sex, race/ethnicity, and stroke type (ischemic vs hemorrhagic) represent the stroke patient population at
Table 1.

Characteristics of participants $(N=39)$.

\begin{tabular}{|c|c|c|}
\hline Variable & Mean \pm SD & Range \\
\hline Age (yr) & $64.54 \pm 14.13$ & 39-94 \\
\hline Admission NIHSS Score & $8.82 \pm 4.84$ & $2-21$ \\
\hline $\begin{array}{l}\text { NIHSS Upper-Limb } \\
\text { Score (Impaired) }\end{array}$ & $1.82 \pm 1.31$ & $0-4$ \\
\hline Premorbid Barthel Score & $99.47 \pm 2.54$ & $85-100$ \\
\hline $\begin{array}{l}\text { Premorbid Modified } \\
\text { Rankin Index }\end{array}$ & $0.38 \pm 0.66$ & $0-2$ \\
\hline & \multicolumn{2}{|c|}{ Number (\%) } \\
\hline \multicolumn{3}{|l|}{ Sex } \\
\hline Male & \multicolumn{2}{|c|}{$17(44)$} \\
\hline Female & \multicolumn{2}{|c|}{$22(56)$} \\
\hline \multicolumn{3}{|l|}{ Race/Ethnicity } \\
\hline Caucasian & \multicolumn{2}{|c|}{$18(46)$} \\
\hline African American & \multicolumn{2}{|c|}{$20(51)$} \\
\hline Other & \multicolumn{2}{|c|}{$1(3)$} \\
\hline \multicolumn{3}{|l|}{ Stroke Type } \\
\hline Ischemic & \multicolumn{2}{|c|}{$29(74)$} \\
\hline Hemorrhagic & \multicolumn{2}{|c|}{$10(26)$} \\
\hline \multicolumn{3}{|l|}{ Affected Side } \\
\hline Dominant & \multicolumn{2}{|c|}{$15(38)$} \\
\hline Nondominant & \multicolumn{2}{|c|}{$24(62)$} \\
\hline NIHSS = National Institute of H & Stroke Scale, SD = & rd deviation. \\
\hline
\end{tabular}

Barnes-Jewish Hospital. Hemorrhagic stroke was defined as having a primary hemorrhage; those subjects judged to have hemorrhagic transformation of an ischemic infarct were classified as having ischemic stroke. The majority of subjects were affected on their nondominant side.

Group mean, median, and range on the functional limitation and disability measures at 90 days poststroke are provided in Table 2. A broad range of ARA test and WMFT scores was found. For the ARA test, subjects in this sample varied from the minimum score of 0 to the maximum scores on each of the four ARA subtests and on the total. For the WMFT functional ability score, no subject attained the minimum score of 0 but 12/39 (31\%) attained the maximum score of 5 . On the disability measures, a broad distribution of scores was also seen with the FIM motor, FIM UL, and MAL scores. In addition to the MAL QOM and AOU scores, we chose to track the number of activities attempted. This number could be important in reflecting disability or changes in disability because scoring of the MAL is based on activities that the subject attempted, leaving out those activities not attempted. 
Table 2.

Participants’ 90-day poststroke scores on upper-limb functional limitation and disability scales $(N=39)$.

\begin{tabular}{lcccc}
\hline \multicolumn{1}{c}{ Variable } & $\begin{array}{c}\text { Maximum } \\
\text { Score }\end{array}$ & $\begin{array}{c}\text { Mean } \pm \text { Standard } \\
\text { Deviation }\end{array}$ & Median & Range \\
\hline Functional Limitation Measures & 57 & $44.03 \pm 16.14$ & 51.5 & $0-57$ \\
ARA Total & 18 & $12.34 \pm 6.37$ & 15.5 & $0-18$ \\
ARA Pinch & 18 & $14.42 \pm 5.50$ & 18.0 & $0-18$ \\
ARA Grasp & 12 & $9.82 \pm 3.52$ & 12.0 & $0-12$ \\
ARA Grip & 9 & $7.76 \pm 2.36$ & 9.0 & $0-9$ \\
ARA Gross Motor & 5 & $4.14 \pm 1.22$ & 4.68 & $0.64-5.00$ \\
WMFT Functional Ability Score & $*$ & $9.08 \pm 16.25$ & 3.75 & $1.27-78.41$ \\
WMFT Time Scale & & & & $40-90$ \\
Disability Measures & 91 & $81.00 \pm 12.98$ & 86.0 & $16-35$ \\
FIM Motor & 35 & $31.36 \pm 4.83$ & 33.0 & $0.87-5.00$ \\
FIM Upper-Limb & 5 & $3.67 \pm 1.06$ & 3.86 & $1.23-5.00$ \\
MAL Quality of Movement & 5 & $3.90 \pm 1.16$ & 4.26 & $0-29$ \\
MAL Amount of Use & 30 & $19.75 \pm 8.98$ & 22.0 & \\
MAL Number of Activities Attempted & & & & \\
\hline
\end{tabular}

*WMFT maximum time allowable $=120 \mathrm{~s}$; lower scores indicate better (faster) performance.

ARA = Action Research Arm (test), FIM = Functional Independence Measure, MAL = Motor Activity Log, WMFT = Wolf Motor Function Test.

We performed a series of $t$-tests to compare scores on study measures by stroke subtype and affected side. Comparisons of hemorrhagic and ischemic stroke subtypes with the use of the Wilcoxon test revealed no significant differences on any measure. Similarly, comparison between dominant and nondominant affected side showed no significant differences on any measure.

In evaluating possible ceiling effects, we found that examination of individual scores revealed that 41 percent (16/39) of participants achieved maximum scores on the ARA test and 36 percent (14/39) of participants achieved maximum scores on the WMFT functional ability scale. To examine whether persons with full recovery on functional limitation scales still had residual disability, we examined FIM and MAL scores for subjects who had perfect or nearperfect scores on the ARA test and WMFT (Table 3). We found that these subjects nonetheless reported residual disability. Moreover, evidence of persistent disability was found in these persons with little measured functional limitation. Inspection of the mean FIM motor and UL scores indicates that a majority of persons in this group are at or near independence. The standard deviation values, however, indicate that measurable disability is present within this group, where 62 percent scored 6 or less on at least one FIM item. The MAL results are more striking. No individual reported doing all 30 common tasks that make up the MAL. More than half of the subjects reported not taking off their shoes or putting a key in a door. Of the MAL tasks performed in everyday life, all subjects with little or no measured functional impairment reported some decreased AOU and quality of performance on at least one activity.

We computed correlation coefficients to examine the relationships among the measures of functional limitation and disability at 90 days. The total ARA test score was correlated with the FIM motor score $(r=0.47)$, MAL QOM score $(r=0.61)$, and MAL number of activities attempted $(r=0.60)$. The total ARA test was more highly correlated with the WMFT time score $(r=-0.65)$ and WMFT functional ability score $(r=0.95)$.

Correlations between the WMFT subscales and other measures at 90 days were also computed. The WMFT functional ability score was highly correlated with the FIM motor score $(r=0.74)$, the MAL QOM score $(r=$ 0.65 ), and the MAL number of activities attempted ( $r=$ 0.79). The correlation was lower for the MAL AOU score $(r=0.40)$. The correlations for the WMFT time score were FIM motor, $r=-0.43$; MAL QOM, $r=-0.43$; and MAL number of activities attempted, $r=-0.63$. The lowest correlation for the WMFT time scale was with the MAL AOU, $r=0.007$. 
JRRD, Volume 43, Number 3, 2006

Table 3.

Self-reported disability scores at 90 days poststroke in participants with little (near-perfect score) or no (perfect score) upper-limb functional limitation.

\begin{tabular}{|c|c|c|c|c|}
\hline Variable & $\begin{array}{c}\text { Maximum } \\
\text { Score }\end{array}$ & $\begin{array}{c}\text { Mean } \pm \text { Standard } \\
\text { Deviation }\end{array}$ & Range & $\begin{array}{l}\% \text { of Subjects at } \\
\text { Maximum Score }\end{array}$ \\
\hline \multicolumn{5}{|l|}{$\overline{\text { ARA }>56(n=16)}$} \\
\hline FIM Motor & 91 & $86.12 \pm 11.02$ & $46-91$ & 38 \\
\hline FIM Upper-Limb & 35 & $33.37 \pm 2.80$ & $24-35$ & 43 \\
\hline MAL Quality of Measure & 5 & $3.93 \pm 1.19$ & $0.15-5.00$ & 13 \\
\hline MAL Amount of Use & 5 & $4.28 \pm 1.04$ & $1.55-5.00$ & 25 \\
\hline \multicolumn{5}{|c|}{ WMFT Functional Ability Score $>4.8(n=14)$} \\
\hline FIM Motor & 91 & $88.36 \pm 3.18$ & $81-91$ & 43 \\
\hline FIM Upper-Limb & 35 & $34.29 \pm 0.99$ & $31-35$ & 57 \\
\hline MAL Quality of Measure & 5 & $4.22 \pm 0.66$ & $3.2-5.0$ & 14 \\
\hline MAL Amount of Use & 5 & $4.17 \pm 1.13$ & $1.55-5.00$ & 36 \\
\hline
\end{tabular}

\section{DISCUSSION}

The proliferation of randomized controlled trials in rehabilitation is forcing a critical examination of optimal trial methodology. One important element in trial design is the selection of appropriate end points. Currently, no consensus exists on the appropriate end points for UL motor intervention trials, and particularly for the inpatient rehabilitation phase of care. One step in developing this consensus will be understanding the relationships between impairment, functional limitation, and disability measures. In short, we are examining the convergent and divergent validity of these measures in this hemiparetic population at 90 days after stroke [28]. The measures reported in this article are among the most commonly used assessments in rehabilitation clinical trials involving ULs. One aim of VECTORS is the examination of candidate trial end points in preparation for a pivotal efficacy trial of CIMT early after stroke onset, and we are reporting the first results from this yet-to-be-completed preliminary trial.

We found that subjects who met our inclusion criteria demonstrated a surprisingly large amount of variability on study measures. Study participants were not selected on the basis of a total score on a clinical assessment, but rather a series of specific UL motor impairments in the absence of moderate or severe sensory or cognitive deficits. Study participants had to be sufficiently affected to justify 2 weeks of inpatient rehabilitation, yet still have some residual volun- tary UL motor function. Even on the baseline assessment of overall stroke severity (NIHSS), scores varied from mild to severe. Later, at the 90-day time point, the same patterns of variability were evident in measures of functional limitation and disability. At 90 days poststroke, subjects are at both extremes on the functional limitation measures. Besides primary motor system dysfunction, the sources of this variability may include conditions such as apraxia, depression, anosodiaphoria [29], and learned nonuse and are beyond the purview of this phase II clinical trial. Another source of variability may stem from differences in self-reported versus measured disability. The use of self-reported disability measures, however, is widespread in most stroke acute intervention trials such as the National Institute of Neurological Disorders and Stroke rt-PA (tissue plasminogen activator) trial [30].

Our results suggest that high scores on the ARA test and the WMFT cannot be used as proxies for full recovery of everyday UL use on the affected side in early rehabilitation treatment trials. In our sample, subjects who appeared fully recovered on these functional limitation measures at 90 days still had some measurable disability. In the group of participants with no detected functional limitations, all reported somewhat diminished QOM on the MAL tasks. In this sample of 39 individuals (selected on criteria likely to be typical of future early UL intervention trials), these functional limitation measures did not capture the full spectrum of motor dysfunction. 
These findings help to further describe the relationships between the measured components of UL motor function and self-reported UL use in real life [8]. Understanding these relationships is important particularly in rehabilitation trials that are designed to improve realworld (and not laboratory) performance. Improvements in functional limitation did not necessarily lead to full recovery of complex ADL. Correlations between the ARA test, WMFT, and MAL scores suggest that, in this sample, improved QOM was not sufficient for substantial everyday UL use.

This study has some limitations. Our findings are relevant to a select group of stroke survivors without moderate or severe cognitive or sensory impairments who were treated at a specific time after stroke and may not apply to a broader sample of stroke patients. Yet, within a sample such as ours, with minimal cognitive and sensory impairments, one might hypothesize that improvements in motor function would have the greatest chance of lessening disability. Our inclusion criteria are likely to be typical of those criteria used for studies of early UL recovery or interventions that require some voluntary movement. The disability scores are self-report scores and may not reflect actual performance.

Others have noted that motor capacity does not necessarily predict actual use [31], and our data suggest that this finding is also relevant in an early UL motor intervention trial. Our data indicate that for an early UL motor intervention study requiring voluntary motor movement after stroke, excellent performance on motor functional limitation measures cannot be assumed to equal excellent everyday UL productive use. We found that participants at the ceiling of the ARA test and WMFT are not reporting full use of the affected UL in common productive activities. Moreover, speed of movement, commonly used to measure motor function, was not associated with self-reported UL use in this sample.

\section{CONCLUSIONS}

Our results indicate that excellent motor recovery as measured by functional limitation and impairment scales did not equal restoration of everyday productive UL use and speed of task completion did not translate to actual use. Our results confirm the need for a measurement strategy that is sensitive to change, assesses a broad range of performance, and detects meaningful clinical improvements in early rehabilitation intervention trials.

\section{ACKNOWLEDGMENTS}

Dr. Dromerick is now with the National Rehabilitation Hospital, Washington, DC.

We wish to thank Jack Baty, Stacy Outman, Brenda Galloy, and Joanne Wagner for their contributions to the VECTORS study.

This material was based on work supported by National Institute for Neurologic Diseases and Stroke, grant NS41261-01, and the James S. McDonnell Foundation, grant 21002032.

The authors have declared that no competing interests exist.

\section{REFERENCES}

1. Gresham GE, Duncan PW, Stason WB. Post-stroke rehabilitation clinical practice guideline, No. 16. Rockville (MD): AHCPR; 1995.

2. Chae J, Fang ZP, Walker M, Pourmehdi D. Intramuscular electromyographically controlled neuromuscular electrical stimulation for upper limb recovery in chronic hemiplegia. Am J Phys Med Rehabil. 2001;80(12):935-41.

[PMID: 11821677]

3. Van der Lee JH, Wagenaar RC, Lankhorst GJ, Vogelaar TW, Deville WL, Bouter LM. Forced use of the upper extremity in chronic stroke patients: Results from a single-blind randomized clinical trial. Stroke. 1999;30(11):2369-75.

[PMID: 10548673]

4. Dromerick AW, Edwards DF, Hahn M. Does the application of constraint-induced movement therapy during acute rehabilitation reduce arm impairment after ischemic stroke? Stroke. 2000;31(12):2984-88. [PMID: 11108760$]$

5. Winstein CJ, Miller JP, Blanton S, Taub E, Uswatte G, Morris DM, Nichols D, Wolf S. Methods for a multisite randomized trial to investigate the effect of constraintinduced movement therapy in improving upper extremity function among adults recovering from a cerebrovascular stroke. Neurorehabil Neural Repair. 2003;17(3):137-52. [PMID: 14503435]

6. Ferraro M, Palazzolo JJ, Krol J, Krebs HI, Hogan N, Volpe BT. Robot-aided sensorimotor arm training improves outcome in patients with chronic stroke. Neurology. 2003; 61(11):1604-7. [PMID: 14663051]

7. Cifu DX, Stewart DG. Factors affecting functional outcome after stroke: A critical review of rehabilitation interventions. Arch Phys Med Rehabil. 1999;80(5 Suppl 1):S35-39.

[PMID: 10326901]

8. Van der Lee JH, Beckerman H, Knol DL, De Vet HC, Bouter LM. Clinimetric properties of the motor activity log 
for the assessment of arm use in hemiparetic patients. Stroke. 2004;35(6):1410-14. [PMID: 15087552]

9. Uswatte G, Taub E. Constraint-induced movement therapy: New approaches to measurement in rehabilitation. In: Stuss DT, Winocur G, Robertson IH, editors. Cognitive neurorehabilitation. Cambridge (England): Cambridge University Press; 1999.

10. Wylie CM. The value of early rehabilitation in stroke. Geriatrics. 1970;25(5):107-13. [PMID: 4909720]

11. Paolucci S, Antonucci G, Grasso MG, Morelli D, Troisi E, Coiro P, Bragoni M. Early versus delayed inpatient stroke rehabilitation: A matched comparison conducted in Italy. Arch Phys Med Rehabil. 2000;81(6):695-700. [PMID: 10857508]

12. Biernaskie J, Chernenko G, Corbett D. Efficacy of rehabilitative experience declines with time after focal ischemic brain injury. J Neurosci. 2004;24(5):1245-54. [PMID: 14762143]

13. Brott T, Marler JR, Olinger CP, Adams HP Jr, Tomsick T, Barsan WG, Biller J, Eberle R, Hertzberg V, Walker M. Measurement of acute cerebral infarction: Lesion size by computed tomography. Stroke. 1989;20(7):871-75. [PMID: 2749847]

14. Poole JL, Whitney SL. Motor assessment scale for stroke patients: concurrent validity and interrater reliability. Arch Phys Med Rehabil. 1988;69(3 Pt 1):195-97. [PMID: 3348720]

15. Katzman R, Brown T, Fuld P, Peck A, Schechter R, Schimmel $\mathrm{H}$. Validation of a short Orientation-Memory-Concentration test of cognitive impairment. Am J Psychiatry. 1983;140(6):734-39. [PMID: 6846631]

16. Mahoney FI, Barthel DW. Functional evaluation: The Barthel Index. Md State Med J. 1965;14:61-65. [PMID: 14258950]

17. Rankin J. Cerebral vascular accidents in patients over the age of 60. II. Prognosis. Scott Med J. 1957;2(5):200-215. [PMID: 13432835]

18. Jorgensen HS, Nakayama H, Raaschou HO, Vive-Larsen J, Stoier M, Olsen TS. Outcome and time course of recovery in stroke. Part II: Time course of recovery. The Copenhagen Stroke Study. Arch Phys Med Rehabil. 1995;76(5): 406-12. [PMID: 7741609]

19. De Weerdt W, Harrison MA. Measuring recovery of armhand function in stroke patients: A comparison of the Brunnstrom-Fugl-Meyer test and the Action Research Arm test. Physiother Can. 1985;37:65-70.
20. Lyle RC. A performance test for assessment of upper limb function in physical rehabilitation treatment and research. Int J Rehabil Res. 1981;4(4):483-92. [PMID: 7333761]

21. Morris DM, Uswatte G, Crago JE, Cook EW 3rd, Taub E. The reliability of the Wolf Motor Function Test for assessing upper extremity function after stroke. Arch Phys Med Rehabil. 2001;82(6):750-55. [PMID: 11387578]

22. Taub E, Miller NE, Novack TA, Cook EW 3rd, Fleming WC, Nepomuceno CS, Connell JS, Crago JE. Technique to improve chronic motor deficit after stroke. Arch Phys Med Rehabil. 1993;74(4):347-54. [PMID: 8466415]

23. Wolf SL, Lecraw DE, Barton LA, Jann BB. Forced use of hemiplegic upper extremities to reverse the effect of learned nonuse among chronic stroke and head-injured patients. Exp Neurol. 1989;104(2):125-32. [PMID: 2707361]

24. Uswatte G, Taub E, Morris DM, Vignolo M, McCulloch K. Reliability and validity of the upper-extremity Motor Activity Log-14 for measuring real-world arm use. Stroke. 2005; 36(11):2493-96. [PMID: 16224078]

25. Granger CV, Hamilton BB, Linacre JM, Heinemann AW, Wright BD. Performance profiles of the functional independence measure. Am J Phys Med Rehabil. 1993; 72(2):84-89. [PMID: 8476548]

26. Granger CV, Hamilton BB, Sherwin FS. Guide for use of the uniform data set for medical rehabilitation. Buffalo (NY): Department of Rehabilitation Medicine, Buffalo General Hospital; 1986.

27. Hall K, Hamilton BB, Gordon WA, Zasler ND. Characteristics and comparisons of functional assessment indices: Disability Rating Scale, Functional Independence Measure, and Functional Assessment Measure. J Head Trauma Rehabil. 1993;8(2):60-74.

28. Campbell DT, Fiske DW. Convergent and discriminate validation by the multitrait-multimethod matrix. Psychol Bull. 1959;56(2):81-105. [PMID: 13634291]

29. Critchley M. Observations on anosodiaphoria. Encephale. 1957;46(5-6):540-46. [PMID: 13501156]

30. The National Institute of Neurological Disorders and Stroke rt-PA Study Group. Tissue plasminogen activator of acute ischemic stroke. New Engl J Med. 1995;333(24): 1581-87. [PMID: 7477192$]$

31. Andrews K, Stewart J. Stroke recovery: He can but does he? Rheumatol Rehabil. 1979;18(1):43-48. [PMID: 424667]

Submitted for publication April 26, 2005. Accepted in revised form April 21, 2006. 